\section{AB0288 PERSISTENTLY ACTIVE DISEASE IN PATIENTS WITH NEW-ONSET RHEUMATOID ARTHRITIS DURING THE FIRST YEAR OF TIGHT CONTROL AND AGGRESSIVE THERAPY: PREVALENCE AND CLINICAL PREDICTORS IN DIFFERENT CRITERIA SETS}

Wenhui Xie, Xiaoying Sun, Zhuoli Zhang. Peking University First Hospital,

Department of Rheumatology and Clinical Immunology, Beijing, China

Background: Early intervention and aggressive therapy of rheumatoid arthritis (RA) improves prognosis, however a substantial proportion of patients under guideline-based care remain persistently active disease $(P A D)$ in the first year

Objectives: To investigate the proportion of PAD (LDA/MDA/HDA) in reallife practice and identify its prognostic factors in early RA ( $\leq 2$ years) patients receiving tight-controlled conventional synthetic disease-modifying antirheumatic drugs (csDMARD) treatment at 1 year of follow-up.

Methods: In our observational RA cohort, patients were usually threemonthly followup and step-up csDMARD escalation treatment at the discretion of physicians in the first year. Disease activity and remission were assessed by four commonly-used indices (DAS28-ESR, CDAI, SDAl, and Boolean criteria). Logistic regression was used to estimate odds ratios (ORs) and their $95 \%$ confidence intervals (Cls) for PAD by 12 months.

Results: The study included 303 DMARD-naïve early RA patients completing one year follow-up schedule, who were mostly female $(72 \%)$ with a mean age of 55 , and a median disease duration of 6 months. Most patients were in MDA or HDA at baseline and most of them $(83.5 \%)$ received csDMARDS combination in the first year. $26.4-55.1 \%$ of participants did not reach remission by 12 months. Compared to patients experienced remission, patients in PAD were frequently in female, elder, shorter disease duration, and high baseline disease activity components, including SJC, TJC, PGA, EGA, ESR, CRP and glucocorticoids use. In multivariate logistic regression analyses, female $(\mathrm{OR}=1.34-1.58)$, increasing age $(\mathrm{OR}=1.03-1.10)$, longer disease duration $(\mathrm{OR}=1.45-1.78)$, higher baseline SJC (OR=1.11-1.25), EGA $(\mathrm{OR}=1.02-1.08)$ showed the independent association with the increased risk for PAD, across all criteria. Higher ESR (OR=1.05) was an independent risk factor for PAD, measured by DAS28-ESR

Conclusion: Our results indicated around one-third of RA participants receiving guideline-based healthcare had PAD in the first year. Female, increasing age, long disease duration, higher SJC, EGA, and ESR at baseline independently increased the risk of PAD.

Disclosure of Interests: None declared

DOI: 10.1136/annrheumdis-2019-eular.947

\section{AB0289 PREDICTION OF CARDIOVASCULAR EVENTS IN RHEU- MATOID ARTHRITIS PATIENTS USING PROTEIN BIO- MARKERS AND CLINICAL FACTORS}

Jeffrey Curtis ${ }^{1}$, Fenglong Xie ${ }^{1}$, Lang Chen ${ }^{1}$, Eric H. Sasso ${ }^{2}$, Elena Hitraya ${ }^{2}$, Jerry Lanchbury ${ }^{3}$, Darl Flake ${ }^{3}$, Alexander Gutin ${ }^{3}$, Cheryl Chin ${ }^{4}$, Cynthia

S. Crowson ${ }^{5}$. ${ }^{1}$ University of Alabama at Birmingham, Birmingham, United States of America; ${ }^{2}$ Crescendo BioScience, Salt Lake City, United States of America;

${ }^{3}$ Myriad, Salt Lake City, United States of America; ${ }^{4}$ Crescendo BioScience, South San Francisco, United States of America; ${ }^{5}$ Mayo Clinic, Rochester, MN, United States of America

Background: The ACC/AHA recommends preventive strategies for patients with a high-predicted risk of atherosclerotic cardiovascular disease (CVD). RA patients are at higher risk for CVD events, yet the role of systemic inflammation and the influence of traditional CVD risk factors are unclear with respect to risk prediction in RA.

Objectives: A simple and accurate algorithm for predicting CVD event risk that considers systemic inflammation might help risk assessment for RA patients and optimize preventive care.

Methods: We derived a U.S. cohort of RA patients by linking multi-biomarker disease activity (MBDA) test data to Medicare claims data Patients had to have $\geq 1$ year Medicare coverage prior to the date of their first MBDA test, which was designated as baseline. Exclusions were past MI, stroke, or cancer. Follow-up ended at the earliest of: 1) CVD event; 2) death; 3) loss of coverage; or 4) 12/31/2016, the latest date for which the Medicare claims database was evaluated. CVD events were defined as incident $\mathrm{MI}$, stroke or fatal CVD event, and were identified using validated algorithms (PPV $>=80 \%$ ). The leptin-adjusted MBDA score (Curtis et al., Rheumatology 2018) and its 12 individual protein biomarkers were evaluated as predictors of CVD events, as were other variables, including demographics, healthcare utilization, CVD-related comorbidities and medications, and RA-related features (e.g. DMARD/biologic use, glucocorticoid use). The cohort was randomly split 2:1 to use
$2 / 3$ of patients for training and $1 / 3$ for validation. Cox proportional hazard regression with LASSO was used for variable selection based on minimization of 10 -fold cross-validated error $+1 \mathrm{SE}$. Model calibration (observed vs. expected) and discrimination were assessed for predicted CVD events at 3 years. Analyses are ongoing; model performance results are reported for the cross-validated training data.

Results: A total of 26,261 eligible RA patients were analyzed; mean (SD) age $68.6(10.2)$ years, $80.1 \%$ female, $72.6 \%$ white, $23 \%$ diabetes, $43 \%$ statin use, $56 \%$ methotrexate, $44 \%$ on biologics/tofacitinib, $55 \%$ steroids, and median (IQR) adjusted MBDA score 40 (32-49). A total of 477 CVD events occurred over mean (SD) follow-up time of 1.7 (1.2) years yielding a CVD incidence rate of $16.5(95 \% \mathrm{Cl} 15.0-18.0) / 1000 \mathrm{py}$. The most important predictors in these LASSO-selected models were age, betablocker use, sex, diabetes, adjusted MBDA score and a subset of individual MBDA biomarkers. The best performing model had a cross-validated area under the receiver operator curve of 0.70 and good observed: expected prediction at 3 years (Figure).

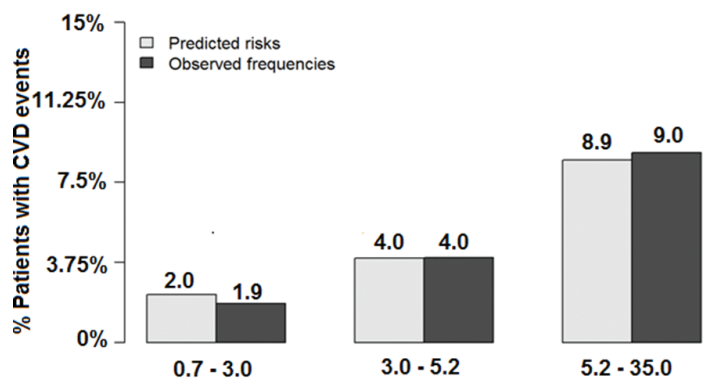

Predicted risk groups

Figure 1

Conclusion: Preliminary results from this approach suggest that a simple algorithm consisting of a limited number of protein biomarkers and clinical measures can provide an accurate method to predict short-term CVD risk in RA.Acknowledgement: The work for this abstract was funded by an industry/academic collaboration between Myriad and University of Alabama at Birmingham.

Disclosure of Interests: Jeffrey Curtis: None declared, Fenglong Xie: None declared, Lang Chen: None declared, Eric H. Sasso Shareholder of: Myriad Genetics, Inc., Employee of: Crescendo Bioscience, Inc., Elena Hitraya Shareholder of: Myriad, Employee of: Myriad, Jerry Lanchbury Shareholder of: Myriad, Employee of: Myriad, Darl Flake Shareholder of: Myriad Genetics, Inc., Employee of: Myriad Genetics, Inc., Alexander Gutin Shareholder of: Myriad, Employee of: Myriad, Cheryl Chin Shareholder of: myriad stock, Employee of: myriad, Cynthia S. Crowson: None declared

DOI: 10.1136/annrheumdis-2019-eular.6323

\section{AB0290 TREAT-TO-TARGET (T2T) IS NOT ENOUGH: IDENTIFY FACTORS LEADING TO A MISMATCH BETWEEN T2T AND HAQ AMONG RA PATIENTS THROUGH DATA MINING FROM SMART SYSTEM OF DISEASE MANAGEMENT (SSDM)}

Jing Yang ${ }^{1}$, Yongfu Wang ${ }^{2}$, Hua Wei ${ }^{3}$, Yu Zhang ${ }^{1}$, Xiaoling Liu ${ }^{4}$, Xiaofeng Rong ${ }^{5}$, Jinmei Zou ${ }^{1}$, Xiaofei Shi ${ }^{6}$, LI Hongbin ${ }^{7}$, Hui Xiao ${ }^{8}$, Yuhua Jia ${ }^{8}$, Fei Xiao ${ }^{8}$, Zhenbiao Wu ${ }^{9}$, SSDM Collaboration Group, China. ${ }^{1}$ Central Hospital of MianYang, MianYang, China; ${ }^{2}$ The First Affiliated Hospital of BaoTou Medical College, BaoTou, China; ${ }^{3}$ Northern Jiangsu People's Hospital, Yangzhou, China; ${ }^{4}$ The 1 st Affiliated Hospital of Guangzhou University of TCM, Guangzhou, China; ${ }^{5}$ The 1 st Affiliated hospital of Chongqing medical University, Chongqing, China; ${ }^{6}$ The First Affiliated Hospital of Henan University of Science and Technology, Luoyang, China; ${ }^{7}$ The Affiliated Hospital of Inner Mongolia Medical University, Hohhot, China; ${ }^{8}$ Shanghai Gothic Internet Technology Co., Ltd., Shanghai, China; ${ }^{9}$ The First Affiliated Hospital of The Fourth Military Medical University, Xi'an, China

Background: T2T, achieving a DAS28 score lower than 2.6 (remission) or below 3.2 (low disease activity), is the main management strategy recommended by ACR and EULAR. HAQ is the most widely used in assessments of physical function in RA. However, the T2T may not always associate with good $\mathrm{HAQ}$.

Objectives: To quantify phenomenon of mismatch between the T2T and the $H A Q$ in RA patients and identify influential factors from real-world data mining in SSDM. 